\title{
Higgs Effective Field Theory
}

\section{José R. Espinosa*}

ICREA, Institució Catalana de Recerca i Estudis Avançats, Barcelona, Spain IFAE, Universitat Autònoma de Barcelona 08193 Bellaterra, Barcelona, Spain

E-mail: espinosalifae.es

This lecture gives a brief introduction to the use of the effective field theory approach in the search of indirect signals of Beyond the Standard Model physics through the study of Higgs phenomenology. After discussing how to best organize the basis of dimension-6 operators (expected to be the dominant ones), we discuss the hierarchy of constraints one can set on these operators already from pre-LHC data and we identify the operators that can only be constrained by LHC Higgs data. Finally, we discuss the constraints from Higgs data and identify the few operators that could still show large deviations in the future.

Proceedings of the Corfu Summer Institute 2015 "School and Workshops on Elementary Particle Physics and Gravity"

1-27 September 2015

Corfu, Greece

${ }^{*}$ Speaker. 


\section{Motivation and goals}

The use of an effective field theory (EFT) approach to the study of beyond the Standard Model (BSM) physics and its searches is well motivated given that the new physics scale $\Lambda$ seems to be heavier than the EW scale (as we learn from negative LHC searches), $\Lambda>O(T e V)>M_{E W}$. Besides the usual good properties of EFTs (see [1] for reviews), the use of EFTs has three other advantages in the present context: 1) it offers a model-independent approach (complementary to the study of particular BSM scenarios well motivated for whatever reason), 2) it is very useful to guide us in what interactions to look for by telling us if a given operator is expected to be more or less suppressed (we will refine our power counting below) and also if it is already constrained by existing data or relatively unconstrained (in which case, it might well be the place to look for a relatively large departure from the SM) and 3) it allows to probe (although indirectly) mass scales above the direct reach of the LHC. This latter point could be crucial (if a deviation is found!) e.g. to decide which next-generation collider to build. With the Higgs discovery and all data LHC has accumulated so far, we are now in the position to look at the complete Lagrangian of dimension-6 operators, $\mathscr{L}_{d=6}$, and (making use of data from previous experiments, in particular LEP I and II and Tevatron) perform a global anaysis to determine which are the most promising deviations from the SM we can expect. As is well known, the Higgs plays a central role in any BSM model that addresses the hierarchy problem. Such BSM physics should talk to the Higgs and, therefore, we focus mostly on Higgs physics as a promising ground for deviations.

\section{2. $\hbar$-Power Counting}

As is well known, $d=6$ operators that violate baryon $(B)$ or lepton $\left(L_{i}\right)$ numbers require a very large suppresion scale while we are interested rather in new physics expected to appear not far from the TeV scale (if naturalness is a good guide). We therefore consider only the $d=6$ operators that respect $B$ and $L_{i}$. Concerning their flavor structure, for the same reason we will assume minimal flavor violation (MFV) holds, up to possible violations for the top quark. Under these assumptions we expect that $d=6$ operators will be suppressed by $1 / \Lambda^{2}$ with $\Lambda \sim$ a few TeV.

Here we want to be more precise and include also an estimate for the effect of couplings in these irrelevant operators. The low-energy impact of a given operator $\mathscr{O}_{i}$ will be very different depending on such effects. Consider e.g. the difference between

$$
\frac{1}{\Lambda^{2}} g_{i}^{2} \mathscr{O}_{i}, \quad \text { vs. } \quad \frac{1}{\Lambda^{2}} g_{i}^{4} \mathscr{O}_{i}, \quad \text { vs. } \quad \frac{1}{\Lambda^{2}} \frac{g_{i}^{2}}{16 \pi^{2}} \mathscr{O}_{i}, \quad\left(\text { with } g_{i} \ll 1\right) .
$$

Such coupling factors depend of course on the particular UV physics that generales the operators but one can make rather generic estimates that are useful to guess the relative importance of different operators in whole classes of BSM theories (e.g. if they are perturbative or if some particular sector couples strongly to the new physics, etc.). First we have to agree on a good notation for couplings (e.g., a quartic coupling $|H|^{2} S^{2}$ of the Higgs to some heavy scalar $S$, we could call $g_{H}^{2}$, or $g_{H}^{4}$, or $\lambda_{H}$ making the task of determining the coupling factors of irrelevant operators ill-defined). The sensible way to do this is the following.

In Quantum Field Theory, the perturbative expansion in powers of interaction couplings is also a loop expansion in powers of $\hbar$ and it is then natural to define couplings in a way that keeps track 
of $\hbar$ powers. As the simplest example take a scalar field $\phi$ with Lagrangian

$$
\mathscr{L}=\frac{1}{2}\left(\partial_{\mu} \phi\right)^{2}-\frac{1}{2} m^{2} \phi^{2}-\frac{1}{4} g^{2} \phi^{4} .
$$

The quantity that appears in the path-integral formulation and controls the perturbative/loop expansion is the exponential of the action (in units of $\hbar$ ):

$$
\frac{i}{\hbar} S=\frac{i}{\hbar} \int d^{4} x \mathscr{L}
$$

By a field redefinition $\phi=\sqrt{\hbar} \hat{\phi}$, the above ratio reads

$$
\frac{i}{\hbar} S=i \int d^{4} x\left[\frac{1}{2}\left(\partial_{\mu} \hat{\phi}\right)^{2}-\frac{1}{2} m^{2} \hat{\phi}^{2}-\frac{1}{4}\left(g^{2} \hbar\right) \hat{\phi}^{4}\right]
$$

which explicitly shows that the perturbative expansion is an expansion in powers of $g^{2} \hbar$. This procedure can be generalized to any number of fields and interaction couplings $g_{i}$. In fact, it is convenient to define all the couplings $g_{i}$ such that $g_{i}^{2} \hbar$ are the perturbative expansion parameters.

Using the same field-rescaling as in the previous example for a generic Lagrangian term

$$
\frac{i}{\hbar} g^{n-2} \phi_{1} \ldots \phi_{n} \quad \rightarrow \quad i\left(g^{2} \hbar\right)^{n / 2-1} \hat{\phi}_{1} \ldots \hat{\phi}_{n}
$$

where $g$ stands for a generic coupling and it is understood that several different couplings might appear, it is immediate to derive the following rule:

$\underline{(n-2) \text { Rule: }}$

an operator in a generic $\mathscr{L}$ containing $n$ fields (irrespective of their spin) should carry $n-2$ powers of couplings.

The couplings in the Standard Model Lagrangian satisfy this prescription, provided we use the counting $y_{f}, g_{\alpha} \sim g$ and $\lambda \sim g^{2}$, where $y_{f}$ are the Yukawa couplings, $g_{\alpha}$ the gauge couplings and $\lambda$ the Higgs quartic coupling.

With this prescription, radiative corrections of $L$-loop order to a given interaction vertex between $n$ fields will automatically carry $n-2+2 L$ powers of couplings: $n-2$ from its tree-level counting and 2 more from each additional loop, that contributes a $g^{2} \hbar /\left(16 \pi^{2}\right)$ factor. To see that this counting works to arbitrary loop order and number of external legs, consider an $L$-loop diagram with a number $V_{i}$ of vertices with $i$-legs each, $I$ internal lines and $E=n$ external legs. According to our rule, each $V_{i}$ vertex introduces $(i-2)$ powers of couplings so that the total power is $\sum_{i}(i-2) V_{i}$. Using the identities $\sum_{i} i V_{i}=2 I+E$ and $I-\sum_{i} V_{i}=L-1$, we get

$$
g^{\sum_{i}(i-2) V_{i}}=g^{n-2+2 L},
$$

as anticipated. Notice that, with this convention for couplings, strong coupling corresponds to $g \sim 4 \pi$, for which all loop corrections are of the same order.

\section{Application to the $d=6 \mathrm{SM}$ effective Lagrangian}

Let us apply the $n-2$ prescription rule discussed in the previous section to the operators of the $d=6 \mathrm{SM}$ effective Lagrangian, revisiting some power counting analyses in recent literature. Notice 
first that the $n-2$ rule is in accord with the generic expansion of the low-energy EFT Lagrangian written as $[2,4]$

$$
\mathscr{L}_{E F T}=\frac{\Lambda^{4}}{g_{*}^{2}} \mathscr{L}\left(\frac{D_{\mu}}{\Lambda}, \frac{g_{H} H}{\Lambda}, \frac{g_{f} \psi}{\Lambda^{3 / 2}}, \frac{g_{\alpha} F_{\mu v}}{\Lambda^{2}}\right),
$$

where $\Lambda$ represents the scale of BSM new physics (assumed to be significantly higher than the EW scale for the EFT expansion to be applicable); $g_{H}, g_{f}$ represent generic couplings of the SM Higgs field and fermions to that BSM sector and $g_{\alpha}$ represent the usual SM gauge couplings with $F_{\mu \nu}$ the SM gauge field-strengths. Finally, $g_{*}$ is a generic coupling that might be different depending on the particular Lagrangian term considered.

Consider next the $d=6$ terms of the effective Lagrangian. After eliminating redundant operators using equations of motion (EoMs), we can use a basis that consist of the following 8 classes of operators:

$$
\left\{g_{\alpha} X^{3}, g_{\alpha}^{2} X^{2} H^{2}, y g_{\alpha} \psi^{2} X H, g_{h}^{4} H^{6}, g_{d h}^{2} D^{2} H^{4}, y g_{y}^{2} \psi^{2} H^{3}, g_{f h}^{2} \psi^{2} H^{2} D, g_{f}^{2} \psi^{4}\right\},
$$

where, following standard practice, $X$ stands for gauge field-strengths and $D$ for covariant derivatives. Although similar bases have been used in the past, the peculiarity of definition (3.2) is the normalization of each class with a given power of couplings in accordance with the $n-2$ prescription (compare e.g. to [5]). ${ }^{1}$ In (3.2) we have explicitly used Yukawa couplings in those operators that involve a chirality flip and have kept a generic gauge coupling $g$ in those operators that involve $X$ 's. We have kept different couplings for $H$ and $\psi$ fields depending also on the type of operator involved. Our generic choices serve as a useful bookkeeping device. In concrete BSM models the UV theory determines what couplings appear in each operator and a more precise assignment becomes possible. Moreover, in many cases different coupling combinations appear within a single operator class. As an example, paying attention to chirality flips we would write $y_{u} y_{d}\left(\bar{Q}_{L}^{r} u_{R}\right) \varepsilon_{r s}\left(\bar{Q}_{L}^{s} d_{R}\right)$, and for this particular 4-fermion operator we have $g_{f}^{2}=y_{u} y_{d}$, while a 4-fermion operator like $g_{f}^{2}\left(\bar{u}_{R} \gamma_{\mu} u_{R}\right)\left(\bar{d}_{R} \gamma^{\mu} d_{R}\right)$ is not expected to carry a Yukawa dependence in $g_{f}^{2}$.

This power counting is useful even when some of the couplings become strong. For instance, if one takes $g_{*} \sim g_{H} \sim g_{f_{L, R}} \sim 4 \pi$, the power counting in (3.1) reproduces the so-called naivedimensional-analysis (NDA) counting, usually written in terms of $\Lambda$ and $f \equiv \Lambda /(4 \pi)$, that gives the following scaling for a generic term of the effective Lagrangian:

$$
\delta \mathscr{L} \sim f^{2} \Lambda^{2}\left(\frac{\phi}{\Lambda}\right)^{A}\left(\frac{\psi}{f \sqrt{\Lambda}}\right)^{B}\left(\frac{g F_{\mu v}}{\Lambda^{2}}\right)^{C}\left(\frac{D_{\mu}}{\Lambda}\right)^{D} .
$$

In discussing $d=6$ operators we find useful to write them as products of currents [3, 4] (like in Fermi's theory) as this makes transparent the connection with potential examples of UV theories that can generate such operators by the tree-level exchange of some heavy particle (be it a scalar, a fermion, or a gauge boson). Obviously, the fact that such operators can be generated that way does not mean they must be, but it is good to know. It is interesting that some operators cannot be written as the product of two currents, although they can be easily generated via loops. We can then classify $d=6$ operators in these two classes: "current-current" (or "tree-level") operators and

\footnotetext{
${ }^{1}$ With this prescription, the 1-loop anomalous dimension matrix that describes operator mixing is automatically of order $g^{2} /\left(16 \pi^{2}\right)$, in contrast with the more complicated coupling dependence in bases that do not follow the $n-2$ rule.
} 

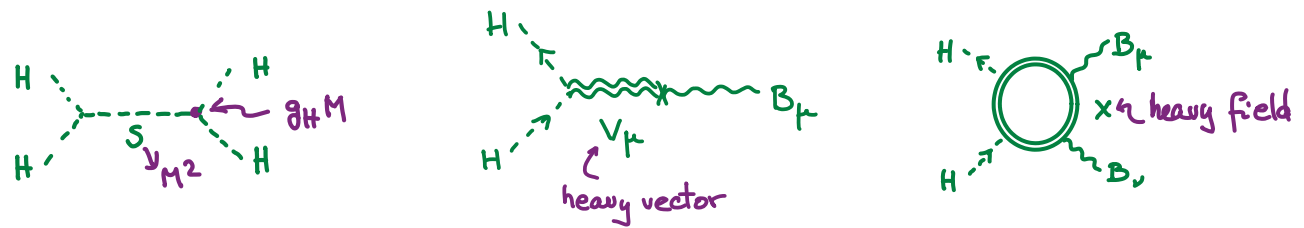

Figure 1: Feynman diagrams with examples of the possible origin of the different types of $d=6$ operators discussed in the text: type 1 (left), type 2 (center) and type 3 (right).

"one-loop" operators. Combining this classification with the power counting discussed above, we find the following three types of $d=6$ operators:

- Type 1: "Current-current" with a potential $g_{*}^{2}$ enhancement, $\delta \mathscr{L}=g_{*}^{2} c_{i} \mathscr{O}_{i} / \Lambda^{2}$. As an example, consider $\mathscr{O}_{H}$ :

$$
\frac{g_{H}^{2}}{\Lambda^{2}} \underbrace{\frac{1}{2}\left(\partial_{\mu}|H|^{2}\right)^{2}}_{\mathscr{O}_{H}}=-\frac{g_{H}^{2}}{2 \Lambda^{2}} \underbrace{|H|^{2}}_{J_{H}} \partial^{2} \underbrace{|H|^{2}}_{J_{H}},
$$

which can be generated by the tree-level exchange of a heavy scalar field coupled to the current $J_{H}=|H|^{2}$. See left diagram in Fig. 1, with the mass $M$ of the heavy scalar to be identified with $\Lambda$.

- Type 2: "Current-current" without $g_{*}^{2}$ enhancement, $\delta \mathscr{L}=c_{i} \mathscr{O}_{i} / \Lambda^{2}$. As an example, consider

$$
\mathscr{O}_{B} \equiv \frac{i g^{\prime}}{2} \underbrace{\left(H^{\dagger}{\left.\stackrel{\leftrightarrow}{D^{\mu}} H\right)}_{J_{B \mu}}^{\partial^{v} B_{\mu v}}\right.}_{J_{H}^{\mu}}
$$

which can be generated by the tree-level exchange of a heavy vector field leading to the product of the indicated currents. See middle diagram in Fig. 1.

- Type 3: "one-loop", $\delta \mathscr{L}=g_{*}^{2} c_{i} \mathscr{O}_{i} /\left(16 \pi^{2} \Lambda^{2}\right)$, like

$$
\mathscr{O}_{B B} \equiv g^{\prime 2}|H|^{2} B_{\mu v} B^{\mu v},
$$

which is straightforward to generate, e.g. by a loop of a heavy field with nonzero hypercharge that also couples to the Higgs. See right diagram in Fig. 1. Obviously, if $g_{*}$ is strong, $g_{*} \sim 4 \pi$, the loop suppresion (expected for weakly coupled theories) will not be present.

\subsection{Operator bases}

The number of $d=6$ operators (that preserve $B$ and $L_{i}$ numbers), first studied systematically in Ref. [6], is 59 (for a single fermion family) [5] 2 : 59 ways to deviate from the SM at order

\footnotetext{
${ }^{2}$ As some of the Wilson coefficients are complex, the number of real parameters describing these operators is higher and goes up to 76 .
} 
$1 / \Lambda^{2}$. Naively one would write more operators but some of them are redundant: they can be eliminated from the Lagrangian by field redefinitions and have no impact on $S$-matrix elements. As an example, take $\mathscr{O}_{r} \equiv|H|^{2}\left|D_{\mu} H\right|^{2}$. It is straightforward to show that the shift $H \rightarrow H-$ $g_{*}^{2} c_{r} H|H|^{2} /\left(2 \Lambda^{2}\right)$ removes $\delta \mathscr{L}=g_{*}^{2} c_{r} \mathscr{O}_{r} / \Lambda^{2}$, and it is known that such field redefinitions do not change the physics. An equivalent way of removing redundant operators is by using $(d=4)$ EoMs on the $d=6$ terms. To see this is allowed, consider a field redefinition $\varphi \rightarrow \varphi+F(\varphi) / \Lambda^{2}$. It changes the action

$$
S=\int d^{4} x\left[\mathscr{L}_{d \leq 4}+\frac{1}{\Lambda^{2}} \mathscr{L}_{d=6}+O\left(\frac{1}{\Lambda^{4}}\right)\right] \rightarrow \delta S=\int d^{4} x\left[\frac{\delta \mathscr{L}_{d \leq 4}}{\delta \varphi} \frac{1}{\Lambda^{2}} F(\varphi)+O\left(\frac{1}{\Lambda^{4}}\right)\right]
$$

so that the $d=6$ Lagrangian shifts as $\mathscr{L}_{d=6} \rightarrow \mathscr{L}_{d=6}+\left(\delta \mathscr{L}_{d \leq 4} / \delta \varphi\right) F(\varphi)$ without changing the physics. Therefore we can drop terms that can be written in the form $\left(\delta \mathscr{L}_{d \leq 4} / \delta \varphi\right) F(\varphi)$ by using $\delta \mathscr{L}_{d \leq 4} / \delta \varphi=0$ which is nothing but the $d=4$ EoM for field $\varphi$.

This point is not just a technicality. It is important because it implies there are different ways of removing redundant operators to arrive at a particular basis of 59 operators. Although physics will not depend on such basis choice, some bases prove more convenient than others (and the literature is plagued with errors due to "wrong" choices of basis) depending of course on the physical effect one is after. The basis I will use appears in the literature under the name "SILH", from Strongly Interacting Light Higgs, as it was chosen as ideally suited to study scenarios of pseudo-Goldstone composite Higgses [7]. Needless to say, it is not restricted to such scenarios and it is as good as any other to parametrize $\mathscr{L}_{d=6}$.

This basis contains 14 CP-even operators made of bosons only, as shown in Table. 1, with the different boxes corresponding to the different types of operators described before, plus the following $6 \mathrm{CP}$-odd operators (3 for $S U(2)_{L}$ and 3 for $S U(3)_{c}$ ):

$$
\begin{aligned}
& \mathscr{O}_{F \tilde{F}}=g_{\alpha}^{2}|H|^{2} F_{\mu \nu}^{\alpha} \tilde{F}^{\alpha \mu v}, \\
& \mathscr{O}_{H \tilde{F}}=i g_{\alpha}\left(D^{\mu} H\right)^{\dagger} T^{\alpha}\left(D^{v} H\right) \tilde{F}_{\mu v}^{\alpha}, \\
& \mathscr{O}_{3 \tilde{F}}=\frac{1}{3} g_{\alpha} f_{\alpha \beta \gamma} \tilde{F}_{\mu}^{\alpha v} F_{v \rho}^{\beta} F^{\gamma \rho \mu} .
\end{aligned}
$$

There are many more operators that involve fermions. I list the 44 of them (for a single family) in Table 2. The total number of operators in this SILH basis is then $14+6+44=64$, five more than the 59 advertised as the number of independent operators. It is at times convenient to live with 5 redundant operators that can be removed from the basis by using EoMs at will, depending on the physics one is interested in.

There are other bases often used in the literature, the most common being the "Hagiwara basis" [8] and the "Polish basis" [5]. The first maximizes the number of operators that involve SM bosons only, without specifying fermion operators. The second tries to minimize the number of operators containing derivatives: it gets rid of many bosonic operators using their EoMs. A comparison of the bosonic operators in these three bases is presented in table 3, taken from ref. [9].

Generically there are two aspects to consider in choosing a good basis, according to the scheme: Theory $\leftrightarrow$ Basis $\leftrightarrow$ Experiment. Ideally, the basis should provide a transparent connection to a theory (or class of theories) so as to minimize the number of operators required to capture the 


\begin{tabular}{|c|}
\hline $\begin{array}{c}\mathscr{O}_{H}=\frac{1}{2}\left(\partial^{\mu}|H|^{2}\right)^{2} \\
\mathscr{O}_{T}=\frac{1}{2}\left(H^{\dagger} \stackrel{\leftrightarrow}{D}_{\mu} H\right)^{2} \\
\mathscr{O}_{6}=\lambda|H|^{6}\end{array}$ \\
\hline \begin{tabular}{c}
$\mathscr{O}_{W}=\frac{i g}{2}\left(H^{\dagger} \sigma^{a} \stackrel{\leftrightarrow}{D^{\mu}} H\right) D^{v} W_{\mu v}^{a}$ \\
$\mathscr{O}_{B}=\frac{i g^{\prime}}{2}\left(H^{\dagger} \stackrel{\leftrightarrow}{D^{\mu}} H\right) \partial^{v} B_{\mu \nu}$ \\
\hdashline $\mathscr{O}_{2 W}=-\frac{1}{2}\left(D^{\mu} W_{\mu \nu}^{a}\right)^{2}-$ \\
$\mathscr{O}_{2 B}=-\frac{1}{2}\left(\partial^{\mu} B_{\mu \nu}\right)^{2}$ \\
$\mathscr{O}_{2 G}=-\frac{1}{2}\left(D^{\mu} G_{\mu \nu}^{A}\right)^{2}$
\end{tabular} \\
\hline 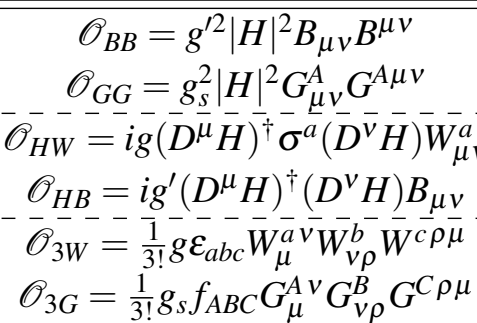 \\
\hline
\end{tabular}

Table 1: 14 CP-even operators made of SM bosons. The operators are grouped in 3 different boxes corresponding to the 3 types of operators discussed in the text (with type 1 at the top, type 2 in the middle and type 3 at the bottom). Dashed lines separate operators of different structure within a given class. There are, in addition, the 6 CP-odd operators given in Eq. (3.8).

physical effects. In addition it is convenient to have a direct connection between operators and the heavy states that produce them and some handle on the size expected for the operators ${ }^{3}$.

As an example, consider BSM theories in which heavy states only couple to SM bosons (the so-called universal theories). The leading $d=6$ physics effects are most conveniently captured by operators containing bosons only. These are the 14 operators (respecting CP) listed in Table 1. This is the number of independent parameters that map the physical effects at $d=6$ level of precision. Now, in some bases several of these operators are removed in favor of other operators using the gauge boson EoMs, e.g.

$$
D^{v} W_{\mu \nu}^{a}=\frac{i g}{2} H^{\dagger} \sigma^{a} \stackrel{\leftrightarrow}{D}_{\mu} H+\frac{g}{2} \sum_{f} \bar{f}_{L} \sigma^{a} \gamma_{\mu} f_{L}
$$

Clearly, such bases are not the best suited ones to study this kind of scenarios: a single physical effect captured by one Wilson coefficient, say $c_{W} \mathscr{O}_{W} / \Lambda^{2}$, in a "good" basis, is spread among many different operators (including all fermions!) in bases that remove $\mathscr{O}_{W}$ by using the EoM above. Moreover, the physical effects controlled by the single parameter $c_{W}$ will appear in the "bad" bases through many Wilson coefficients that are correlated in a very precise way. More explicitly, one has

$$
c_{W} \mathscr{O}_{W} \rightarrow \frac{g^{2}}{g_{*}^{2}} c_{W}\left[-\frac{3}{2} \mathscr{O}_{H}+2 \mathscr{O}_{6}+\frac{1}{2}\left(\mathscr{O}_{y_{u}}+\mathscr{O}_{y_{d}}+\mathscr{O}_{y_{e}}+\text { h.c. }\right)+\frac{1}{4} \sum_{f} \mathscr{O}_{L}^{(3) f}\right] .
$$

\footnotetext{
${ }^{3}$ These kind of arguments are called "theory biased" in some quarters. A better name would be "theory informed".
} 


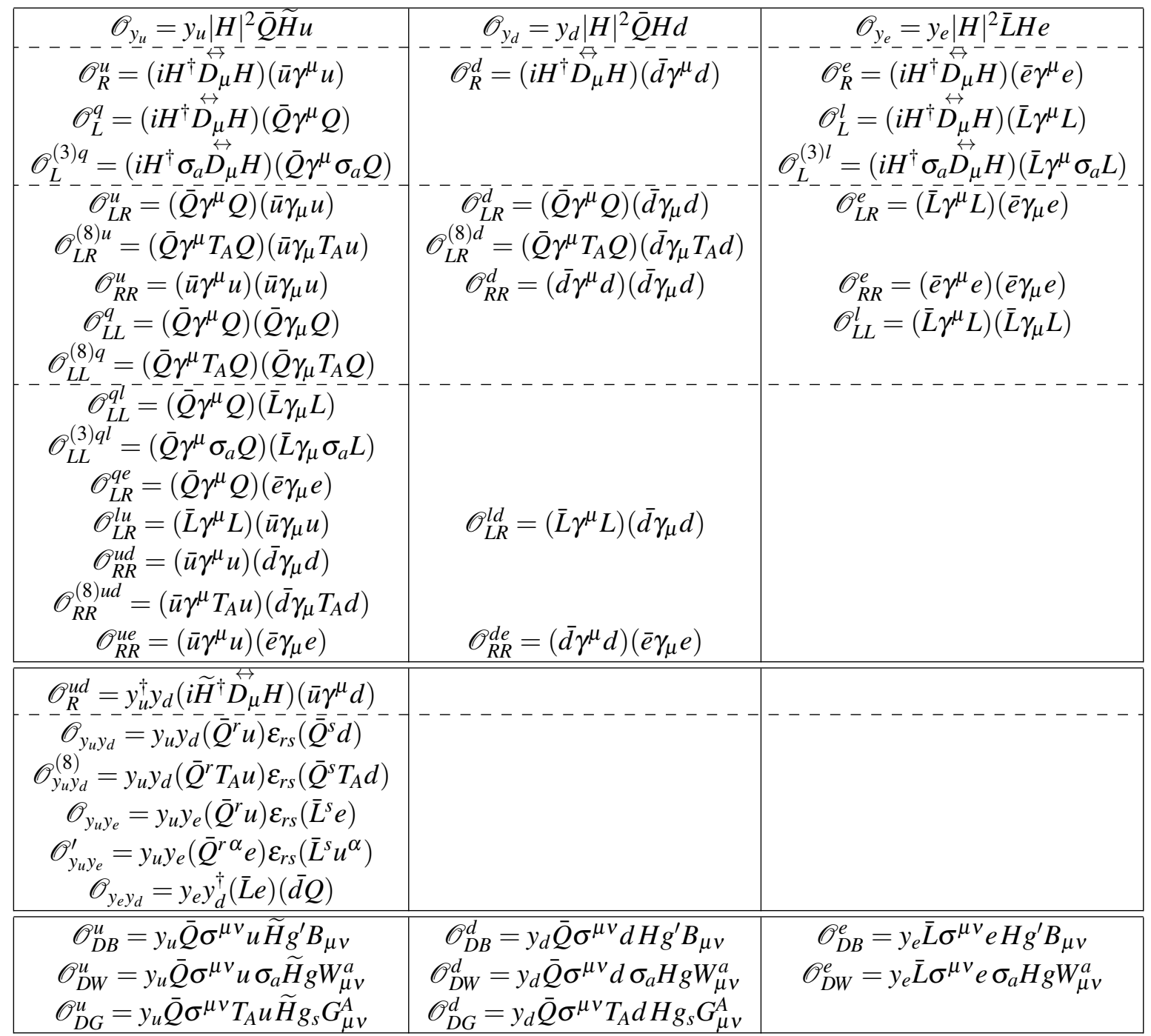

Table 2: 44 operators made of one-family of SM fermions. In the first column there are operators made of the up-type quark and other fermions; in the second column there are operators made only of the down-type quark and leptons; the third column lists operators made only of leptons. The operators are grouped in 3 different boxes corresponding to the 3 classes of operators defined in the text (same order as in Table 1). Dashed lines separate operators of different structure within a given class.

Such potential correlations can be rather misleading on the other front that determines the good properties of a particular basis: its connection to experimental data. In any case, one can easily switch from basis to basis, and tools have been developed to assist in such basis translations, see Ref. [10].

\subsection{Experimental Constraints}

A good basis should have a clean connection between operators and observables, ideally in a one-to-one correspondence, which is however not possible in practice: a given experimental constraint generically involves some linear combination of Wilson coefficients. Extracting model- 


\begin{tabular}{|c|c|c|}
\hline Polish basis & Hagiwara basis & SILH basis \\
\hline $\mathscr{O}_{W}=\varepsilon^{I J K} W_{\mu}^{I v} W_{v}^{J \rho} W_{\rho}^{K \mu}$ & $\mathscr{O}_{W W W}=\operatorname{Tr}\left[\hat{W}_{\mu \nu} \hat{W}^{v \rho} \hat{W}_{\rho}^{\mu}\right]$ & $\mathscr{O}_{3 W}=\frac{1}{3 !} g \varepsilon_{a b c} W_{\mu}^{a v} W_{v \rho}^{b} W^{c \rho \mu}$ \\
\hline $\mathscr{O}_{\varphi W}=\varphi^{\dagger} \varphi W_{\mu \nu}^{I} W^{I \mu \nu}$ & $\mathscr{O}_{W W}=\Phi^{\dagger} \hat{W}_{\mu v} \hat{W}^{\mu v} \Phi$ & - \\
\hline $\mathscr{O}_{\varphi B}=\varphi^{\dagger} \varphi B_{\mu v} B^{\mu v}$ & $\mathscr{O}_{B B}=\Phi^{\dagger} \hat{B}_{\mu v} \hat{B}^{\mu v} \Phi$ & $\mathscr{O}_{B B}=g^{\prime 2}|H|^{2} B_{\mu v} B^{\mu v}$ \\
\hline $\mathscr{O}_{\varphi W B}=\varphi^{\dagger} \sigma^{I} \varphi W_{\mu v}^{I} B^{\mu v}$ & $\mathscr{O}_{B W}=\Phi^{\dagger} \hat{B}_{\mu v} \hat{W}^{\mu v} \Phi$ & - \\
\hline- & $\mathscr{O}_{W}=\left(D_{\mu} \Phi\right)^{\dagger} \hat{W}^{\mu v}\left(D_{v} \Phi\right)$ & $\mathscr{O}_{H W}=i g\left(D^{\mu} H\right)^{\dagger} \sigma^{a}\left(D^{v} H\right) W_{\mu v}^{a}$ \\
\hline- & $\mathscr{O}_{B}=\left(D_{\mu} \Phi\right)^{\dagger} \hat{B}^{\mu v}\left(D_{v} \Phi\right)$ & $\mathscr{O}_{H B}=i g^{\prime}\left(D^{\mu} H\right)^{\dagger}\left(D^{v} H\right) B_{\mu \nu}$ \\
\hline- & $\mathscr{O}_{D W}=\operatorname{Tr}\left(\left[D_{\mu}, \hat{W}_{v \rho}\right]\left[D^{\mu}, \hat{W}^{v \rho}\right]\right)$ & $\mathscr{O}_{2 W}=-\frac{1}{2}\left(D^{\mu} W_{\mu \nu}^{a}\right)^{2}$ \\
\hline- & $\mathscr{O}_{D B}=-\frac{g^{\prime 2}}{2}\left(\partial_{\mu} B_{v \rho}\right)\left(\partial^{\mu} B^{v \rho}\right)$ & $\mathscr{O}_{2 B}=-\frac{1}{2}\left(\partial^{\mu} B_{\mu \nu}\right)^{2}$ \\
\hline - & - & $\mathscr{O}_{W}=\frac{i g}{2}\left(H^{\dagger} \sigma^{a} \overleftrightarrow{D^{\mu}} \mu\right) D^{\nu} W_{\mu \nu}^{a}$ \\
\hline- & - & $\mathscr{O}_{B}=\frac{i g^{\prime}}{2}\left(H^{\dagger} \overleftrightarrow{D}^{\mu} H\right) \partial^{v} B_{\mu v}$ \\
\hline $\mathscr{O}_{\varphi D}=\left(\varphi^{\dagger} D^{\mu} \varphi\right)^{*}\left(\varphi^{\dagger} D_{\mu} \varphi\right)$ & $\mathscr{O}_{\Phi, 1}=\left(D_{\mu} \Phi\right)^{\dagger} \Phi \Phi^{\dagger}\left(D^{\mu} \Phi\right)$ & $\mathscr{O}_{T}=\frac{1}{2}\left(H^{\dagger} \overleftrightarrow{D}_{\mu} H\right)^{2}$ \\
\hline $\mathscr{O}_{\varphi \square}=\left(\varphi^{\dagger} \varphi\right) \square\left(\varphi^{\dagger} \varphi\right)$ & $\mathscr{O}_{\Phi, 2}=\frac{1}{2} \partial_{\mu}\left(\Phi^{\dagger} \Phi\right) \partial^{\mu}\left(\Phi^{\dagger} \Phi\right)$ & $\mathscr{O}_{H}=\frac{1}{2}\left(\partial^{\mu}|H|^{2}\right)^{2}$ \\
\hline $\mathscr{O}_{\varphi}=\left(\varphi^{\dagger} \varphi\right)^{3}$ & $\mathscr{O}_{\Phi, 3}=\frac{1}{3}\left(\Phi^{\dagger} \Phi\right)^{3}$ & $\mathscr{O}_{6}=\lambda|H|^{6}$ \\
\hline- & $\mathscr{O}_{\Phi, 4}=\left(D_{\mu} \Phi\right)^{\dagger}\left(D^{\mu} \Phi\right)\left(\Phi^{\dagger} \Phi\right)$ & - \\
\hline
\end{tabular}

Table 3: Comparison of $C P$-even bosonic operators between three popular bases. (Taken from [9].)

independent bounds on the Wilson coefficients of $d=6$ operators requires using a complete basis and paying special attention to possible hidden correlations. The common practice of setting bounds on a single coefficient at a time ignores correlations (that can be due to theory or simply to a basis choice!), overestimates the experimental constraints and should be avoided. On the other hand, concrete BSM scenarios predict particular patterns of correlations between Wilson coefficients, allowing for more stringent experimental constraints on them. Finally, it is clear that in order to set constraints from experiment on the $c_{i}$ 's, one does not care about their possible enhancement (by $g_{*}^{2}$ ) or suppression [by $g_{*}^{2} /\left(16 \pi^{2}\right)$ ] which only plays a role on the theory $\leftrightarrow$ operators connection.

One could proceed by making a global complete fit of all data for the 59 Wilson coefficients, or the relevant subset for Higgs physics, which is the sector where significant deviations are well motivated. Alternatively $[4,11]$, one can save effort by noticing the hierarchical pattern of experimental constraints (on $c_{i} m_{W}^{2} / \Lambda^{2}$ ) that range from the very precise per-mille level (e.g. from EW precision data) to percent level [e.g. from Triple Gauge Coupling (TGC) data] to loose constraints of order $10 \%$ or less (e.g. for some Higgs couplings). We can imagine pictorially such bounds as an ellipsoid in the space of Wilson coefficients with a hierarchy in the size of its axes. A good basis should be well aligned with such ellipsoid so that a well defined subset of operators can be constrained at the per-mille level (and therefore can be dropped safely from the discussion of the constraints on lesser constrained operators) and so on towards less constrained operators, until one can determine the most promising ones to expect possible large deviations. 


$$
\begin{gathered}
\mathscr{O}_{H}=\frac{1}{2}\left(\partial^{\mu}|H|^{2}\right)^{2} \\
\mathscr{O}_{T}=\frac{1}{2}\left(H^{\dagger} \stackrel{\leftrightarrow}{D}_{\mu} H\right)^{2} \\
\mathscr{O}_{6}=\lambda|H|^{6} \\
\mathscr{O}_{W}=\frac{i g}{2}\left(H^{\dagger} \sigma^{a} \stackrel{\leftrightarrow}{D^{\mu}} H\right) D^{v} W_{\mu v}^{a} \\
\mathscr{O}_{B}=\frac{i g^{\prime}}{2}\left(H^{\dagger} \stackrel{\leftrightarrow}{D^{\mu}} H\right) \partial^{v} B_{\mu v}
\end{gathered}
$$

\begin{tabular}{c}
$\mathscr{O}_{B B}=g^{2}|H|^{2} B_{\mu v} B^{\mu v}$ \\
$\mathscr{O}_{G G}=g_{s}^{2}|H|^{2} G_{\mu \nu}^{A} G^{A \mu \nu}$ \\
$\mathscr{O}_{H W}=i g\left(D^{\mu} H\right)^{\dagger} \sigma^{a}\left(D^{v} H\right) W_{\mu v}^{a}$ \\
$\mathscr{O}_{H B}=i g^{\prime}\left(D^{\mu} H\right)^{\dagger}\left(D^{v} H\right) B_{\mu \nu}$ \\
$\mathscr{O}_{3 W}=\frac{1}{3 !} g \varepsilon_{a b c} W_{\mu}^{a v} W_{v \rho}^{b} W^{c \rho \mu}$ \\
\hline
\end{tabular}

$$
\begin{array}{|c|c|c|}
\hline \mathscr{O}_{y_{u}}=y_{u}|H|^{2} \bar{Q} \widetilde{H} u & \mathscr{O}_{y_{d}}=y_{d}|H|^{2} \bar{Q} H d & \mathscr{O}_{y_{e}}=y_{e}|H|^{2} \bar{L} H e \\
\hdashline \mathscr{O}_{R}^{u}=\left(i H^{\dagger} D_{\mu} H\right)\left(\bar{u} \gamma^{\mu} u\right) & \mathscr{O}_{R}^{d}=\left(i H^{\dagger} D_{\mu} H\right)\left(\bar{d} \gamma^{\mu} d\right) & \mathscr{O}_{R}^{e}=\left(i H^{\dagger} D_{\mu} H\right)\left(\bar{e} \gamma^{\mu} e\right) \\
\mathscr{O}_{L}^{q}=\left(i H^{\dagger} \stackrel{\leftrightarrow}{\mu}_{\mu} H\right)\left(\bar{Q} \gamma^{\mu} Q\right) & & \\
\mathscr{O}_{L}^{(3) q}=\left(i H^{\dagger} \sigma^{a} \stackrel{\leftrightarrow}{D_{\mu}} H\right)\left(\bar{Q} \gamma^{\mu} \sigma^{a} Q\right) & & \\
\mathscr{O}_{L L}^{(3) q l}=\left(\bar{Q} \gamma^{\mu} \sigma^{a} Q\right)\left(\bar{L} \gamma_{\mu} \sigma^{a} L\right) & & \mathscr{O}_{L L}^{(3) l}=\left(\bar{L} \gamma^{\mu} \sigma^{a} L\right)\left(\bar{L} \gamma_{\mu} \sigma^{a} L\right) \\
\hline
\end{array}
$$

Table 4: The $20 d=6$ operators of our basis relevant for Higgs physics. $\mathscr{O}_{L L}^{(3) l}$ has an indirect effect through its modification of Fermi's constant $G_{F} . \mathscr{O}_{L L}^{(3) q l}$ is important for combined experimental constraints (through $G_{F}^{q}$, Fermi's constant measured with quarks).

\subsection{Higgs Physics}

Such program has been carried through, with a focus on Higgs physics, in refs. [11, 12] (where you can find a detailed discussion). Here I will sketch the procedure and main points. The starting point (under the assumptions of MFV) is the subset of 20 operators in Table 4 below, which are the only ones directly relevant to Higgs physics. If these operators are present, they will modify the couplings of the Higgs with respect to its SM values, offering potential windows to new physics. I have indicated above several such effects, some of which have already started to be probed (for the first time!) by the LHC, while others will be in the future. Which ones can still show some large deviation from the SM? First, one should realize that many of these operators have an impact on other sectors of the theory not involving explicitly Higgses being produced, that is, with $H \rightarrow(0, v / \sqrt{2})^{T}$. A famous example is $\mathscr{O}_{T}$, which changes the $m_{W} \leftrightarrow m_{Z}$ relationship. Other operators modify the couplings of fermions to gauge bosons [e.g. $\mathscr{O}_{R}^{f}=\left(i H^{\dagger} \stackrel{\leftrightarrow}{D}_{\mu} H\right)\left(\bar{f} \gamma^{\mu} f\right)$ ] and others triple gauge-boson couplings, like $\mathscr{O}_{H B}=i g^{\prime}\left(D^{\mu} H\right)^{\dagger}\left(D^{v} H\right) B_{\mu \nu}$. One can then use experimental data from LEP + Tevatron to set constraints in many of these operators even before looking at Higgs data.

As we mentioned already, working at orden $1 / \Lambda^{2}$, the expected deviations in observables of interest will be some linear combinations of Wilson coefficients of the $d=6$ operators in the Table 4. We can now be more explicit about what it means in practice to have an operator basis well aligned with experimental observables. The linear system relating observables $q_{i}$ and Wilson coeficientes $c_{i}$ in the SILH (sub)basis above takes the schematic form: 


$$
\left[\begin{array}{c}
q_{i}^{l} \\
q_{i}^{q} \\
q_{i}^{T G C}
\end{array}\right]=\left[\begin{array}{ccc}
T_{1} & 0 & 0 \\
X_{12} & T_{2} & 0 \\
X_{13} & X_{23} & T_{3}
\end{array}\right] \cdot\left[\begin{array}{c}
c_{i}^{a} \\
c_{i}^{b} \\
c_{i}^{c}
\end{array}\right] .
$$

The observables $q_{i}^{l}$ are the very precise $Z$-pole observables measurad at LEP-I (the leptonic $Z$ widths into $v v, l_{L} l_{L}, l_{R} l_{R}$ ) and the $W$ mass (from Tevatron). Deviations in these 4 observables depend, through the matrix $T_{1}$, on just 4 Wilson coefficients, the $c_{i}^{a}$ s: $\left(c_{r}, c_{R}^{e}, c_{L L}^{(3) l}\right.$ and $\left.c_{W}+c_{B}\right)$ which can then be bounded at per-mille level. This makes $X_{12}$ and $X_{13}$ irrelevant for the analysis of less constrained observables. Next come quark observables $q_{i}^{q}\left(\delta \Gamma_{Z}^{\text {had }}, R_{b}, A_{c}, A_{b}\right)$ also well measured by LEP plus $G_{F}^{q} / G_{F}^{l}$ (from KLOE and $\beta$ decays) which depend, through $T_{2}$, on just 5 additional coefficients, the $c_{i}^{b}$ 's: $\left(c_{L}^{q}, c_{L}^{(3) q}, c_{R}^{u}, c_{R}^{d}, c_{L L}^{(3) q}\right)$ which can in turn be bound at a similar level of precision $\left[c_{i} m_{W}^{2} / \Lambda^{2} \lesssim O\left(10^{-3}\right)\right]$ and make $X_{23}$ irrelevant for what follows. Finally, LEP-II data on TGCs give information on the 3 parameters $g_{1}^{Z}, \kappa_{\gamma}, \lambda_{\gamma}$ and this, through $T_{3}$, can be used to bound the $c_{I}^{c}$ s: ( $\kappa_{3 W}$, and two linear combinations of $c_{W}, \kappa_{H W}$ and $\kappa_{H B}$ ) at the per-cent level.

One concludes that previous data closes $4+5+3=12$ of the 20 possible windows for new physics from which we started. Let us focus then on the 8 operators that remain. They are

$$
\begin{gathered}
\mathscr{O}_{H}=\frac{1}{2}\left(\partial_{\mu}|H|^{2}\right)^{2}, \quad \mathscr{O}_{6}=\lambda|H|^{6}, \quad \mathscr{O}_{y_{f}}=y_{f}|H|^{2} \bar{f}_{L} H f_{R}, \\
\mathscr{O}_{B B}=g^{\prime 2}|H|^{2} B_{\mu \nu} B^{\mu \nu}, \quad \mathscr{O}_{G G}=g_{S}^{2}|H|^{2} G_{\mu \nu}^{A} G^{A \mu \nu},
\end{gathered}
$$

and a linear combination of $\mathscr{O}_{W}, \mathscr{O}_{B}, \mathscr{O}_{H W}$ and $\mathscr{O}_{H B}$ that can be written as

$$
\mathscr{O}_{W W}=g^{2}|H|^{2} W_{\mu \nu}^{a} W^{a \mu \nu},
$$

(an operator not in the basis we used). Using the previous bounds on the $c_{i}$ 's, it turns out that the unconstrained combination associated with this operator is $\kappa_{H W}-\kappa_{H B}$.

What these 8 operators have in common is that they involve $|H|^{2}$, in such a way that replacing $H \rightarrow\langle H\rangle$ just gives operators already in $\mathscr{L}_{d=4}$ and their impact is not observable. This means that only Higgs data can constrain these operators. More precisely,

- $\mathscr{O}_{H}$ modifies the Higgs propagator and therefore changes in a universal way all Higgs couplings (leaving then all Higgs BRs the same).

- $\mathscr{O}_{6}$ will impact $h^{*} \rightarrow h h$, not accesible in the near future.

- $\mathscr{O}_{y_{b}}, \mathscr{O}_{y_{\tau}}$ affect $B R(h \rightarrow b \bar{b})$ and $B R(h \rightarrow \tau \bar{\tau})$, respectively.

- $\mathscr{O}_{y_{t}}$ impacts $B R(h \rightarrow \gamma \gamma)$ by changing the SM top-loop contribution. In addition, it changes the rate of $h t \bar{t}$ associated-production.

- $\mathscr{O}_{B B}$ modifies directly $B R(h \rightarrow Z \gamma)$.

- $\mathscr{O}_{G G}$ changes $\sigma(G G \rightarrow h)$, the main Higgs production mechanism.

- $\kappa_{H W}-\kappa_{H B}$ affects $B R(h \rightarrow \gamma \gamma)$. 
Performing a global fit to all Higgs data from ATLAS and CMS the only significant bounds apply to the Wilson coefficients that contribute directly to SM loop-suppressed processes. At 95\% C.L. one gets [11]

$$
\begin{aligned}
& \frac{m_{W}^{2}}{\Lambda^{2}} \kappa_{G G} \in(-0.8,0.8) \times 10^{-3}, \\
& \frac{m_{W}^{2}}{\Lambda^{2}} \kappa_{B B} \in(-1.3,1.8) \times 10^{-3}, \\
& \frac{m_{W}^{2}}{\Lambda^{2}} \kappa_{Z \gamma} \in(-0.6,1.2) \times 10^{-2},
\end{aligned}
$$

where $\kappa_{Z \gamma}=-\left(\kappa_{H W}-\kappa_{H B}\right) / 4-2 s_{W}^{2} \kappa_{B B}$. Notice that the last bound is possible even though the decay $h \rightarrow Z \gamma$ has not been seen yet: the experimental limit on its rate is about $10 \times \sigma_{S M}$.

The analysis reviewed above is useful to bound possible deviations from new physics in all sorts of Higgs measurements. As an example, consider the test of custodial symmetry based on measuring

$$
\lambda_{W Z}^{2}-1=\frac{\Gamma(h \rightarrow W W)}{\Gamma^{S M}(h \rightarrow W W)} \frac{\Gamma^{S M}(h \rightarrow Z Z)}{\Gamma(h \rightarrow Z Z)}-1,
$$

which is constrained experimentally to the range $\lambda_{W Z}-1 \in(-0.5,0.1)$ at $95 \%$ C.L. Using the $d=6$ EFT approach one gets

$$
\begin{aligned}
\lambda_{W Z}^{2}-1 & \simeq s_{W}^{2}\left(0.9 c_{W}-2.6 c_{B}+3 \kappa_{H W}-3.9 \kappa_{H B}\right) \frac{m_{W}^{2}}{\Lambda^{2}} \\
& \simeq\left(0.6 \delta g_{1}^{Z}-0.5 \delta \kappa_{\gamma}-1.6 \kappa_{Z \gamma}\right) \frac{m_{W}^{2}}{\Lambda^{2}},
\end{aligned}
$$

and using the bounds on these deviations from TGC and $h \rightarrow Z \gamma$ data, one gets the stronger bound

$$
\lambda_{W Z}^{2}-1 \in(-6,8) \times 10^{-2} .
$$

\section{Conclusions}

The close scrutiny of Higgs properties is of the utmost importance as they provide well motivated windows to probe BSM physics indirectly. I have described in this lecture how the modelindependent EFT approach through $d=6$ operators can be very powerful for this task ${ }^{4}$. Although there is still room for some deviations, we have seen that the number of "open windows" is smaller than naively thought. Be it through indirect signals of BSM or by direct production of new particles (as hinted by the recent diphoton excess in ATLAS and CMS [14]) we expect that the second LHC will be a very productive one!

\section{References}

[1] G. P. Lepage, "What is renormalization?," [hep-ph/0506330];

H. Georgi, "Effective field theory," Ann. Rev. Nucl. Part. Sci. 43 (1993) 209;

\footnotetext{
${ }^{4}$ This is a very active field of research and new developments have appeared since I delivered my lecture, see
} Refs. [13]. 
A. G. Cohen, "Selected topics in effective field theories for particle physics," In *Boulder 1993, Proceedings, The building blocks of creation* 53-100;

D. B. Kaplan, "Effective field theories," [nucl-th/9506035]; "Five lectures on effective field theory," [nucl-th/0510023 ]; "Lectures on effective field theory," http://www.ictp-saifr.org/wp-content/uploads/2015/05/Kaplan1.pdf;

I. Z. Rothstein, "TASI lectures on effective field theories," [hep-ph/0308266];

A. V. Manohar, "Effective field theories," Lect. Notes Phys. 479 (1997) 311 [hep-ph/9606222 ] ;

C. P. Burgess, "Introduction to Effective Field Theory," Ann. Rev. Nucl. Part. Sci. 57 (2007) 329

[hep-th/0701053];

A. Wulzer, "On Effective Field Theories and NDA,"

http://www.pd.infn.it/ wulzer/Teaching/Strongly_coupled_2013/Chapter_4/

I. Stewart, "Effective Field Theory", MIT on-line course

http://ocw.mit.edu/courses/physics/8-851-effective-field-theory-spring-2013/

[2] A. G. Cohen, D. B. Kaplan and A. E. Nelson, "Counting 4 pis in strongly coupled supersymmetry," Phys. Lett. B 412 (1997) 301 [hep-ph/9706275].

[3] J. Elias-Miró, J. R. Espinosa, E. Masso and A. Pomarol, "Renormalization of dimension-six operators relevant for the Higgs decays $h \rightarrow \gamma \gamma, \gamma Z$," JHEP 1308 (2013) 033 [hep-ph/1302.5661] .

[4] J. Elias-Miró, J. R. Espinosa, E. Masso and A. Pomarol, "Higgs windows to new physics through d=6 operators: constraints and one-loop anomalous dimensions," JHEP 1311 (2013) 066 [hep-ph/1308.1879].

[5] B. Grzadkowski, M. Iskrzynski, M. Misiak and J. Rosiek, "Dimension-Six Terms in the Standard Model Lagrangian,” JHEP 1010 (2010) 085 [ hep-ph/1008 . 4884 ] .

[6] W. Buchmuller and D. Wyler, "Effective Lagrangian Analysis of New Interactions and Flavor Conservation,” Nucl. Phys. B 268 (1986) 621.

[7] G. F. Giudice, C. Grojean, A. Pomarol and R. Rattazzi, “The Strongly-Interacting Light Higgs,” JHEP 0706 (2007) 045 [hep-ph/ 0703164 ] .

[8] K. Hagiwara, S. Ishihara, R. Szalapski and D. Zeppenfeld, "Low-energy constraints on electroweak three gauge boson couplings," Phys. Lett. B 283 (1992) 353; "Low-energy effects of new interactions in the electroweak boson sector,” Phys. Rev. D 48 (1993) 2182.

[9] S. Willenbrock and C. Zhang, "Effective Field Theory Beyond the Standard Model," Ann. Rev. Nucl. Part. Sci. 64 (2014) 83 [hep-ph/1401.0470].

[10] A. Falkowski, B. Fuks, K. Mawatari, K. Mimasu, F. Riva and V. sanz, "Rosetta: an operator basis translator for Standard Model effective field theory,” Eur. Phys. J. C 75 (2015) no.12, 583 [hep-ph/1508.05895].

[11] A. Pomarol and F. Riva, "Towards the Ultimate SM Fit to Close in on Higgs Physics," JHEP 1401 (2014) $151[$ hep-ph/1308.2803].

[12] R. S. Gupta, A. Pomarol and F. Riva, "BSM Primary Effects," Phys. Rev. D 91 (2015) no.3, 035001 [hep-ph/1405.0181].

[13] D. Liu, A. Pomarol, R. Rattazzi and F. Riva, "Patterns of Strong Coupling for LHC Searches," [hep-ph/1603.03064];

R. Contino, A. Falkowski, F. Goertz, C. Grojean and F. Riva, "On the Validity of the Effective Field Theory Approach to SM Precision Tests," [hep-ph/1604.06444]. 
[14] ATLAS-CONF-2015-081, "Search for resonances decaying to photon pairs in $3.2 \mathrm{fb}^{-1}$ of $p p$ collisions at $\sqrt{s}=13 \mathrm{TeV}$ with the ATLAS detector"; CMS PAS EXO-15-004 "Search for new physics in high mass diphoton events in proton-proton collisions at $13 \mathrm{TeV}$ ". 